Aim of the study: The BRAF inhibitor vemurafenib has improved progression-free survival and overall surviva in patients with BRAFV600-mutationpositive metastatic melanoma. Here we present the results of an open-label safety study with vemurafenib in patients with metastatic melanoma enrolled in Polish oncological centres. Material and methods: Patients with untreated or previously treated Stage IIIC/IV BRAFV600 mutation-positive melanoma were treated with oral vemurafenib in an initial dose of $960 \mathrm{mg}$ twice daily. Assessments for safety and efficacy were made every 28 days. For the survival analysis the Kaplan-Meier estimator was used with the log-rank tests for bivariate comparisons.

Results: In total, 75 Polish patients were enrolled in the safety study across four centres. At data cut-off 28 patients died (37\%), mainly (26) due to disease progression; 33 (44\%) patients continued vemurafenib after disease progression. The objective response rate was $46 \%$, including two patients with a complete response and 29 with a partial response. Median progression-free survival was 7.4 months. The one-year overall survival rate was $61.9 \%$ (median overall survival was not reached). Seventy-three (97.3\%) patients reported adverse events (AEs), and grade 3-5 toxicity was reported in $49.4 \%$ (37) patients. The most common AEs were: skin lesions (including rash and photosensitivity), arthralgia, and fatigue.

Conclusions: The overall safety profile and response rate of vemurafenib were comparable to those reported in previous studies of this drug. Our study confirmed the value of well-established prognostic features for overall survival, such as initial LDH (lactate dehydrogenase) level and AJCC staging.

Key words: melanoma, metastatic, vemurafenib, BRAF inhibition.

Contemp Oncol (Pozn) 2014; 19 (4): 280-283 DOI: $10.5114 /$ wo.2015.54082

\section{The outcomes of Polish} patients with advanced BRAFpositive melanoma treated with vemurafenib in a safety clinical trial

\author{
Piotr Rutkowski ${ }^{1}$, Katarzyna Kozak ${ }^{1}$, Jacek Mackiewicz², \\ Krzysztof Krzemieniecki ${ }^{3}$, Sergiusz Nawrocki ${ }^{4}$, Ewa Wasilewska-Teśluk ${ }^{5}$, \\ Łukasz Kwinta ${ }^{6}$, Piotr Wysocki ${ }^{7}$, Hanna Koseła-Paterczyk ${ }^{1}$, Tomasz Świtaj ${ }^{1}$
}

${ }^{1}$ Department of Soft Tissue/Bone Sarcoma and Melanoma, Maria Sklodowska-Curie Memorial Cancer Centre and Institute of Oncology, Warsaw, Poland

${ }^{2}$ Chair of Medical Biotechnology, University of Medical Sciences, Poznan, Poland Department of Diagnostics and Cancer Immunology, Greater Poland Cancer Centre, Poznan, Poland Medical Oncology Department, Malgorzata Medical Centre, Śrem, Poland Medpolonia sp. z o.o., Poznan, Poland

${ }^{3}$ Department of Clinical Oncology, Krakow University Hospital, Krakow, Poland ${ }^{4}$ Division of Radiotherapy and Oncology, Department of Clinical Oncology, Silesian Medical University, Katowice, Poland

${ }^{5}$ Department of Oncology, University of Warmia and Mazury, Olsztyn, Poland ${ }^{6}$ Department of Chemotherapy, Greater Poland Cancer Centre, Poznan, Poland 'Department of Clinical Oncology, West Cancer Centre, Szczecin, Poland

\section{Introduction}

BRAF inhibitors are a currently the standard of care in the treatment of patients with advanced melanomas harbouring the BRAF V600 mutation. Vemurafenib was the first molecular targeting agent acting on the mutated BRAF kinase, which demonstrated improvement of overall survival (OS) in a phase III randomised trial. In the study comparing vemurafenib and dacarbazine in untreated stage IV and unresectable stage IIIC patients with BRAF-mutated melanomas, vemurafenib demonstrated rapid objective responses in approximately $50 \%$ of cases, but control of the tumour growth was observed in up to $90 \%$ of cases [1]. Progression-free survival (PFS) was 6.9 months in the vemurafenib arm versus 1.6 months in the dacarbazine arm, and median OS was 13.6 months [1, 2]. The results of this study led to worldwide approval of vemurafenib for the therapy of advanced BRAF-mutated disease. Recently in a large safety study with more than 3000 patients, the safety profile and efficacy of vemurafenib were confirmed in real-world clinical practice [3]. As the general results of this open-label, multicentre study have been already published, we report here the detailed outcomes of patient cohorts treated in Polish centres only.

\section{Material and methods}

In this open-label study (MO25515, NCT01307397) 75 patients from four Polish oncological centres were included to assess the safety and efficacy of vemurafenib in advanced (unresectable stage IIIC or stage IV) melanoma harbouring BRAF V600 mutation confirmed with the cobas 4800 BRAF V600 Mutation Test (Roche Molecular Systems, Branchburg, NJ, USA). The eligible patients were treated with oral vemurafenib in an initial dose of $960 \mathrm{mg}$ twice daily until disease progression (treatment after progression was allowed after a joint decision by the investigator and the sponsor), unacceptable toxicity, consent withdrawal, or death [3]. The rules for dose modifications and interruptions were described previously [3]. The patient 
characteristics are presented in Table 1. Thirty-six (48\%) patients underwent prior systemic therapy (including 6 patients after ipilimumab failure). None of the patients was previously treated with BRAF or MEK inhibitor. Seven patients (9.3\%) had brain metastases (stable, asymptomatic after previous local therapy) at the time of therapy start. The primary end-point of the trial was safety. Patients were assessed every 28 days. The secondary variables comprised objective response (evaluated by investigators using Response Evaluation Criteria in Solid Tumours RECIST version 1.1), progression-free survival (PFS, calculated from the date of start of vemurafenib therapy to date of progression or death), and overall survival (OS, calculated from the date of start of vemurafenib therapy to the date of death, living patients were censored). For survival analysis the Kaplan-Meier estimator was used with the log-rank tests for bivariate comparisons. Statistical analyses were done with SAS (version 9.2) software.

\section{Results}

All 75 of the enrolled patients started therapy with vemurafenib from October 2011 to June 2012. We present interim analysis prepared on 28 July 2014 with a cut-off date on 31 Jan 2013. Forty-two (56\%) discontinued therapy, with 33 due to progressive disease, one due to adverse events, and three due to death.

The best confirmed responses were as follows: two patients (3\%) had complete response, 29 (43\%) partial response, 30 (45\%) stable disease, and six (8\%) had disease progression. Median duration of response was 7.4 months (95\% Cl: 5.7-9.2). Only eight patients received further systemic therapy (chemotherapy only) after discontinuation of vemurafenib.

Median PFS time was 7.4 months (95\% Cl: 5.5-9.2), and one-year PFS rate was $26.5 \%$ (95\% Cl: 15.3-37.8). Progres-
Table 1. Patients' characteristics $(n=75)$

$\begin{array}{lc}\text { Subgroup } & \boldsymbol{N}(\%) \\ \text { Gender } & \\ \quad \text { Male } & 39(52.0) \\ \text { Female } & 36(48.0) \\ \text { Age (median, years) } 53 & \\ \text { Age < } 65 \text { years } & 65(86.7) \\ \text { Age } \geq 65 \text { years } & 10(13.3) \\ \text { ECOG 0-1 at baseline ECOG } \geq 2 \text { at baseline } & 72(96.0) \\ & 3(4.0) \\ \text { Normal LDH at baseline/screening } & 43(57.3) \\ \text { Elevated LDH at baseline/screening } & 32(42.7) \\ \text { Patients with brain metastases at baseline } & 7(9.3) \\ \text { Patients without brain metastases at baseline } & 68(90.7) \\ \text { Prior Ipilimumab } & 6(8.0) \\ \text { No prior Ipilimumab } & 69(92.0)\end{array}$

sion-free survival significantly correlated with initial lactate dehydrogenase level (median PFS in the LDH normal subgroup was 10.9 months [95\% Cl: $7.5-$ ] vs. 3.8 months [95\% Cl: 3.6-7.2] in the LDH elevated subgroup) (Fig. 1), and the presence of brain metastases at baseline (median PFS was 3.7 months [95\% Cl: 3.6-9.3] in the brain metastases subgroup vs. 7.5 months [ $95 \% \mathrm{Cl}: 5.6-10.3$ ] in patients without brain metastases). For M1 stage subgroups median PFS times were as follows: 7.4 months (95\% Cl: 1.4-) in the M1a subgroup, 10.9 months (6.9-) in the M1b subgroup, and 7.4 (95\% Cl: 4.6-8.3) in the M1c subgroup.

Median OS was not reached; the one-year OS rate was 61.9\% (95\% Cl: 50.1-73.6). OS significantly correlated with initial lactate dehydrogenase level (the one-year OS rate was $85.1 \%$ [74.0-96.1] in the subgroup with normal LDH and $31.4 \%$ [95\% Cl: 14.0-48.9] in the subgroup with elevated LDH [Fig. 2]), and M1 stage (one-year OS rate was $77.8 \%$ [95\% Cl: 50.6-104.9] in the Mla subgroup, 75.0\% [95\% Cl:

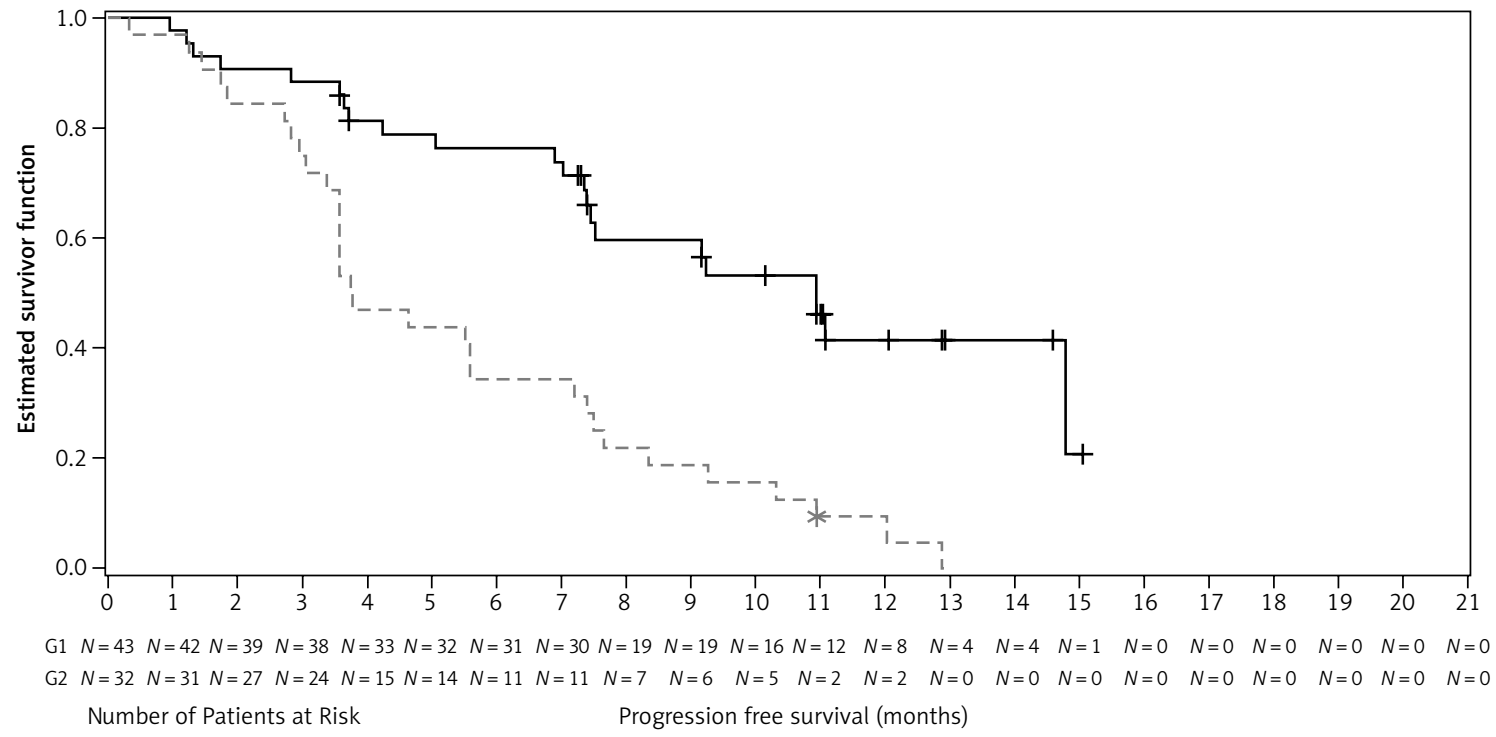

$$
\begin{array}{ll}
\text { — Normal LDH at baseline/screening } & ++ \text { Censored: normal LDH }
\end{array}
$$

Fig. 1. PFS depending on LDH level at the treatment start 


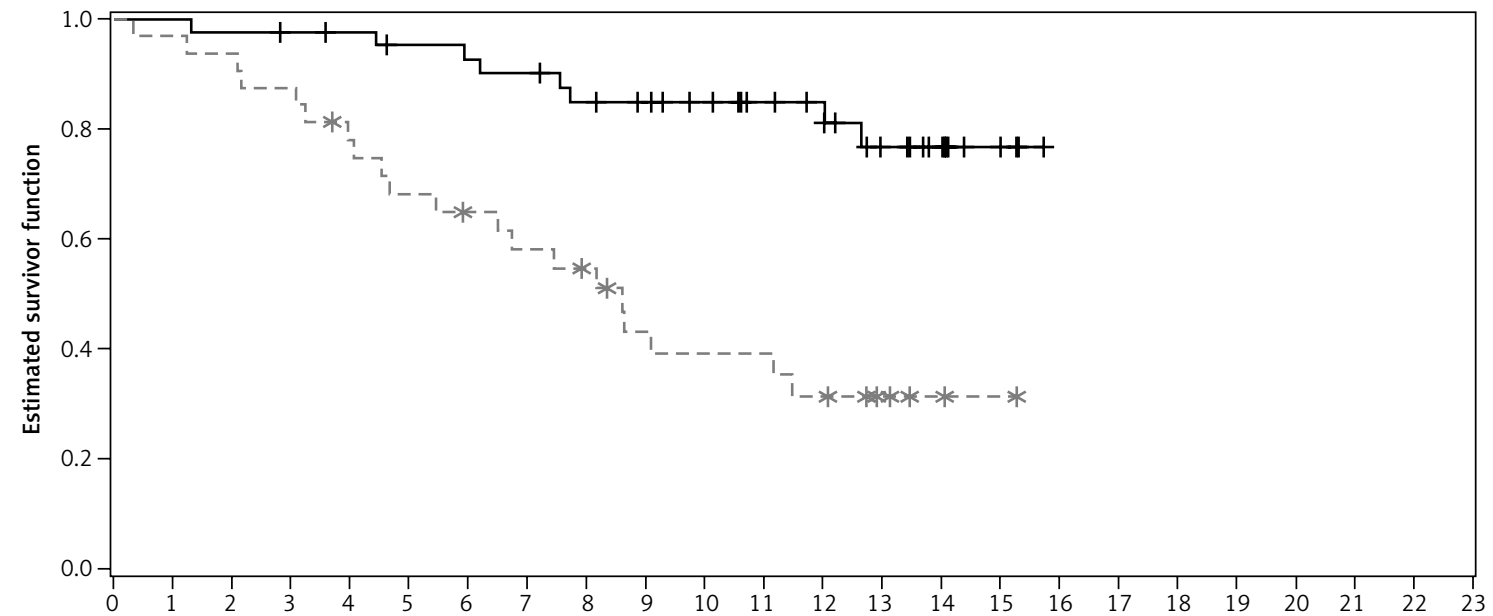

G1 $N=43 N=43 N=42 N=41 \quad N=40 N=38 N=37 N=36 \quad N=33 N=31 \quad N=28 N=24 N=22 N=15 \quad N=11 \quad N=5 \quad N=0 \quad N=0 \quad N=0 \quad N=0 \quad N=0 \quad N=0 \quad N=0 \quad N=0$ G2 $N=32 N=31 N=30 N=28 N=24 N=21 \quad N=19 N=17 \quad N=15 N=11 N=10 N=10 \quad N=8 \quad N=5 \quad N=2 \quad N=1 \quad N=0 \quad N=0 \quad N=0 \quad N=0 \quad N=0 \quad N=0 \quad N=0 \quad N=0$

Number of Patients at Risk

$$
\text { — Normal LDH at baseline/screening }
$$
Time (months)

Fig. 2. OS depending on LDH level at the treatment start

$$
\begin{aligned}
& +\quad+\text { Censored: normal LDH } \\
& * * \text { Censored: elevated LDH }
\end{aligned}
$$

\begin{tabular}{|c|c|c|}
\hline Adverse event & $\begin{array}{c}\text { Grade } 3 \\
(N=75) \\
n(\%)\end{array}$ & $\begin{array}{c}\text { Grade } 4 \\
(N=75) \\
n(\%)\end{array}$ \\
\hline Number of patients with at least one AE & $35(46.7)$ & 0 \\
\hline $\begin{array}{l}\text { Neoplasms benign, malignant and } \\
\text { unspecified } \\
\text { keratoacanthoma } \\
\text { papilloma } \\
\text { squamous cell carcinoma of skin } \\
\text { malignant melanoma } \\
\text { melanocytic naevus } \\
\text { skin papilloma } \\
\text { basal cell carcinoma } \\
\text { fibrous histiocytoma } \\
\text { seborrhoeic keratosis }\end{array}$ & $\begin{array}{l}18(24.0) \\
5(6.7) \\
2(2.7) \\
3(4.0) \\
2(2.7) \\
2(2.7) \\
2(2.7) \\
1(1.3) \\
1(1.3) \\
1(1.3)\end{array}$ & $\begin{array}{l}0 \\
0 \\
0 \\
0 \\
0 \\
0 \\
0 \\
0 \\
0\end{array}$ \\
\hline $\begin{array}{l}\text { Skin and subcutaneous tissue disorders } \\
\text { acanthosis } \\
\text { rash } \\
\text { hyperkeratosis }\end{array}$ & $\begin{array}{l}10(13.3) \\
5(6.7) \\
5(6.7) \\
1(1.3)\end{array}$ & $\begin{array}{l}0 \\
0 \\
0 \\
0\end{array}$ \\
\hline $\begin{array}{l}\text { Investigations } \\
\text { alanine aminotransferase increased } \\
\text { aspartate aminotransferase increased } \\
\text { blood alkaline phosphatase increased } \\
\text { electrocardiogram qt prolonged } \\
\text { hyponatraemia }\end{array}$ & $\begin{array}{l}6(8.0) \\
2(2.7) \\
2(2.7) \\
2(2.7) \\
1(1.3) \\
3(4.0)\end{array}$ & $\begin{array}{l}0 \\
0 \\
0 \\
0 \\
0 \\
0\end{array}$ \\
\hline $\begin{array}{l}\text { Blood and lymphatic system disorders } \\
\text { anaemia } \\
\text { neutropaenia }\end{array}$ & $\begin{array}{l}4(5.3) \\
3(4.0) \\
1(1.3)\end{array}$ & $\begin{array}{l}0 \\
0 \\
0\end{array}$ \\
\hline $\begin{array}{l}\text { Cardiac disorders } \\
\text { angina unstable } \\
\text { myocardial infarction } \\
\text { hypertension }\end{array}$ & $\begin{array}{l}4(5.3) \\
1(1.3) \\
1(1.3) \\
3(4.0)\end{array}$ & $\begin{array}{l}0 \\
0 \\
0 \\
0\end{array}$ \\
\hline Fatigue & $1(1.3)$ & 0 \\
\hline Arthralgia & $1(1.3)$ & 0 \\
\hline Convulsion & $1(1.3)$ & 0 \\
\hline Urinary tract obstruction & $1(1.3)$ & 0 \\
\hline
\end{tabular}

Table 2. Adverse events grades 3 and 4 by system organ class and preferred term
45-105] in the M1b subgroup, and 57\% [95\% Cl: 42.9-71.0] in the M1c subgroup). The presence of asymptomatic brain metastases, stable after previous therapy at baseline, had no significant impact on OS.

At the time of data cut-off 28 patients died (37\%), mainly (26) due to disease progression. Thirty-three (44\%) patients continued vemurafenib after disease progression.

Due to adverse events (AEs) 44 patients (57\%) needed at least one dose modification (reduction or interruption), and 28 patients (37\%) needed dose reduction. 97.3\% (73) of patients had at least one $A E$, and grade 3-5 toxicity was reported in $49.4 \%$ (37) patients. The most common AEs were as previously reported: skin lesions (including rash and photosensitivity), arthralgia, and fatigue. Table 2 summarises AEs occurring in at least $10 \%$ of patients, and Table 3 shows AEs at grade $3-4$. Eight patients (10.7\%) developed cutaneous squamous cell carcinoma (cuSCC), and two - new primary melanomas.

\section{Discussion}

The constitutive hyperactivation of the RAS/RAF/MEK/ ERK pathway (also termed the Mitogen Activated Protein Kinase - MAPK pathway) has been identified in the majority of melanomas as the critical player in the regulation of cell proliferation, invasion, and survival $[1,2,4]$. In 50-70\% of cases this genetic background is achieved via oncogenic mutation of the BRAF gene, mainly V600E point mutation. Vemurafenib is the first BRAF inhibitor class agent approved worldwide for therapy of advanced BRAF-mutated melanoma, and has demonstrated improvement in OS and PFS as compared to dacarbazine. Thereafter, BRAF inhibitors (vemurafenib and dabrafenib) have become the worldwide standard of care for the majority of BRAF-mutant metastatic melanomas (especially established in patients with high tumour burden or progression after immunotherapy). The data presented in our analysis confirm other long-term follow-up studies demonstrating 
median PFS more than six months, and approximately one fourth of patients were still alive after a longer period of time [2, 5]. However, the future of monotherapy with BRAF inhibitors is limited due to the relatively short duration of response in the majority of patients. The addition of a MEK inhibitor to a BRAF inhibitor enhances inhibition of tumour growth and delays acquired resistance [6]. The results from recently published three phase III studies provide convincing evidence that combination therapy with either vemurafenib and cobimetinib or dabrafenib and trametinib will become a new standard treatment for advanced BRAF-mutant melanoma. These randomised trials report median progression-free survival ranging from 9.3 to 11.4 months in combination-therapy groups. In addition, higher response rates (64-68\%) have been achieved with no additional overall toxicity [7-9].

Nevertheless, the current study demonstrates on a national level the efficacy of vemurafenib monotherapy in a group of patients with advanced melanoma with eligibility criteria similar to routine practice and requirements for reimbursement of vemurafenib therapy in Poland. The overall survival data from this study are very encouraging, especially taking into account that almost $50 \%$ of the patients were treated with previous systemic therapy. Moreover, our study confirmed the value of well-established prognostic features for overall survival such as initial LDH level and AJCC staging $[10,11]$. The presence of stable brain metastases had no impact on overall survival in our patients, which confirms the activity of vemurafenib for controlling brain lesions and implies that the mechanism of disease progression is equally related to the lesions outside the central nervous system [12]. The fact that vemurafenib was continued in $44 \%$ of patients after objective disease progression may also have a positive impact on survival [13].

The safety profile observed was consistent with that reported in previous studies, with cutaneous manifestations, arthralgia, and fatigue as the most common AEs. The number of patients with grade 3 or 4 treatment-related adverse events was up to $50 \%$, but only two patients discontinued therapy due to unacceptable toxicity. Photosensitivity (related probably to the chemical structure of the vemurafenib molecule, not to BRAF inhibition per se) is commonly reported during vemurafenib therapy, whereas pyrexia (characteristic for dabrafenib) was rarely noted. cuSCC occurred in approximately $11 \%$ of patients, and their pathogenesis can be related to paradoxical activation of the MAPK pathway in BRAF wild-type cells during selective BRAF V600 mutant kinase inhibitor therapy. Keratoproliferative lesions often require additional surgery, and their frequency implies careful skin examinations every four weeks during vemurafenib therapy.

To summarise, our analysis confirms the efficacy of vemurafenib in a large group of Polish patients treated within the frames of a treatment-use clinical trial. The adverse events were manageable, and the number of cuSCCs was even lower than in phase II-III clinical trials [4, 14]. The understanding of the mechanisms of resistance to BRAF inhibitors monotherapy and development of combination strategy for overcoming early disease progression is the most important issue for the immediate future in the therapy of advanced melanoma.

F. Hoffmann-La Roche provided funding for the study and statistical analyses.

The authors declare no conflict of interest.

\section{References}

1. Chapman PB, Hauschild A, Robert C, et al. Improved survival with vemurafenib in melanoma with BRAF V600E mutation. N Engl J Med 2011; 364: 2507-16.

2. McArthur GA, Chapman PB, Robert C, et al. Safety and efficacy of vemurafenib in BRAF(V600E) and BRAF(V600K) mutation-positive melanoma (BRIM-3): extended follow-up of a phase 3, randomised, open-label study. Lancet Oncol 2014; 15: 323-32.

3. Larkin J, Del Vecchio M, Ascierto PA, et al. Vemurafenib in patients with BRAF(V600) mutated metastatic melanoma: an open-label, multicentre, safety study. Lancet Oncol 2014; 15: 436-44.

4. Shaw HM, Nathan PD. Vemurafenib in melanoma. Expert Rev Anticancer Ther 2013; 13: 513-22.

5. Flaherty L, Hamid O, Linette G, et al. A single-arm, open-label, expanded access study of vemurafenib in patients with metastatic melanoma in the United States. Cancer J 2014; 20: 18-24.

6. Ribas A, Gonzalez R, Pavlick A, et al. Combination of vemurafenib and cobimetinib in patients with advanced BRAF(V600)-mutated melanoma: a phase 1b study. Lancet Oncol 2014; 15: 954-965.

7. Larkin J, Ascierto PA, Dréno B, et al. Combined vemurafenib and cobimetinib in BRAF-mutated melanoma. N Engl J Med 2014; 371: 1867-76.

8. Long GV, Stroyakov-Skiy D, Gogas H, et al. Combined BRAF and MEK inhibition versus BRAF inhibition alone in melanoma. N Engl J Med 2014; 371: 1877-88.

9. Robert C, Karaszewska B, Schachter J, et al. Improved overall survival in melanoma with combined dabrafenib and trametinib. N Engl J Med 2015; 372: 30-9.

10. Balch CM, Gershenwald JE, Soong SJ, et al. Final version of 2009 AJCC melanoma staging and classification. J Clin Oncol 2009; 27: 6199-6206.

11. Cybulska-Stopa B, Skoczek M, Ziobro M, et al. Results of systemic treatment of cutaneous melanoma in inoperable stage III and IV. Contemp Oncol (Pozn) 2012; 16: 532-45.

12. Dummer R, Goldinger SM, Turtschi CP, et al. Vemurafenib in patients with BRAF(V600) mutation-positive melanoma with symptomatic brain metastases: final results of an open-label pilot study. Eur J Cancer 2014; 50: 611-21.

13. Chan MM, Haydu LE, Menzies AM, et al. The nature and management of metastatic melanoma after progression on BRAF inhibitors: effects of extended BRAF inhibition. Cancer 2014; 120: 3142-53.

14. Sosman JA, Kim KB, Schuchter L, et al. Survival in BRAF V600-mutant advanced melanoma treated with vemurafenib. N Engl J Med 2012; 366: 707-14.

\section{Address for correspondence}

\section{Katarzyna Kozak}

Department of Soft Tissue/Bone Sarcoma and Melanoma

Maria Sklodowska-Curie Memorial

Cancer Centre and Institute of Oncology

Roentgena 5

02-781 Warsaw, Poland

e-mail:wiater.katarzyna@gmail.com

Submitted: 22.02 .2015

Accepted: 20.07 .2015 